\title{
The Effect of Stroke on Pharyngeal Laterality During Swallowing
}

\author{
Seoyon Yang, MD, Kyoung Hyo Choi, MD, PhD, Yu Ri Son, MD
}

Department of Rehabilitation Medicine, Asan Medical Center, University of Ulsan College of Medicine, Seoul, Korea

Objective To investigate whether patterns of dysphagia and swallowing laterality differ according to the location of brain lesions in patients with stroke.

Methods Patients with stroke $>20$ years of age were enrolled in this study. A videofluoroscopic swallowing study (VFSS) including the anterior-posterior view was used to assess swallowing. Patterns of swallowing were classified into three types according to the width of barium sulfate flow while passing the pharyngoesophageal segment: right-side-dominant flow, left-side-dominant flow, and no laterality in flow. Laterality was defined when the width of one side was twice or more the width of the other side.

Results A total of 92 patients who underwent swallowing function evaluations by VFSS were enrolled from September 2012 to May 2013. Of these, 72 patients had supratentorial lesions (group I) and 20 patients had infratentorial lesions (group II). Only 10 patients (13.9\%) in group I and three patients (15.0\%) in group II showed laterality. Of these 13 patients, laterality occurred on the left side regardless of the side of the brain lesion. No relationships were found between swallowing laterality and location of stroke or motor weakness.

Conclusion The results suggest that swallowing laterality was not prevalent among patients with stroke and that lesion side, location of the brain lesion, or motor weakness did not influence swallowing laterality. Although stroke can cause symptoms of dysphagia, it is difficult to conclude that stroke has a crucial impact on swallowing laterality.

Keywords Deglutition disorders, Deglutition, Stroke

\section{INTRODUCTION}

Patients with stroke involving the cerebral hemispheres

Received June 23, 2014; Accepted November 12, 2014

Corresponding author: Kyoung Hyo Choi

Department of Rehabilitation Medicine, Asan Medical Center University of Ulsan College of Medicine, 88 Olympic-ro 43-gil, Songpa-gu, Seoul 138-736, Korea

Tel: +82-2-3010-3800, Fax: +82-2-3010-6964, E-mail: kyounghyochoi@ gmail.com

(c) This is an open-access article distributed under the terms of the Creative Commons Attribution Non-Commercial License (http://creativecommons. org/licenses/by-nc/4.0) which permits unrestricted noncommercial use distribution, and reproduction in any medium, provided the original work is properly cited.

Copyright $\odot 2015$ by Korean Academy of Rehabilitation Medicine or brain stem often suffer from dysphagia, with an incidence of $30 \%-80 \%$ [1-4]. Dysphagia is a disorder of deglutition that affects oral, pharyngeal, and esophageal phases of swallowing [5]. Interruption of voluntary control of mastication, bolus transport, and impairment of facial, lip, or tongue motor control are caused by cerebral, cerebellar, or brain stem strokes [6] and can result in impairment of swallowing physiology [7]. Dysphagia should be treated, as it can lead to health-related complications, such as pneumonia as well as nutrition and hydration deficits [7-10].

Several tests, such as the clinical bedside swallowing assessment, can be used to evaluate the presence of dys- 
phagia. Among these tests, a videofluoroscopic swallowing study (VFSS) is a standard diagnostic tool for detecting dysphagia [11] and provides information on bolus passage or residue, laryngeal penetration or aspiration, and the overall swallowing mechanism $[7,12]$.

Swallowing laterality has been studied but the exact mechanism underlying this condition remains unknown. Patterns of swallowing have been investigated in healthy subjects [13]. In this study, $35 \%$ of subjects showed left dominant flow, $7 \%$ showed right dominant flow, and the remaining subjects had no laterality in flow. This study showed that even healthy subjects can have a certain degree of swallowing laterality.

In a previous study [14] using functional magnetic resonance imaging (MRI), swallowing was shown to activate the primary motor cortex, primary somatosensory cortex, supplementary motor cortex, prefrontal cortex, transverse temporal gyrus, insular cortex, internal capsule, cingulate gyrus, speech areas, auditory association area, and sensory-motor integration areas. Lateralization in the right hemisphere was greater than in the left and the left-hemisphere was dominant for swallowing tasks.

Whether laterality exists in normal subjects and in patients with stroke remains under debate. Previous studies have attempted to determine whether laterality exists among patients with stroke and to elucidate the value of compensatory techniques, such as head rotation or head tilt, in patients with laterality. In a study involving patients with hemiplegic stroke and dysphagia, swallowing laterality was not significantly related to the severity of dysphagia nor the direction of hemiplegia [15]. In this study, patients with infratentorial lesions were excluded and swallowing laterality of the patients with infratentorial lesions was not studied.

As dysphagic patterns that occur after stroke can be different according to the location of the brain lesion [16], lesion location may influence the direction and laterality of the swallowing pattern. Therefore, whether laterality exists and differs between patients with supratentorial or infratentorial lesions should be assessed.

The aim of this study was to investigate whether patterns of dysphagia and swallowing laterality differ according to the location of brain lesions. Therefore, we classified our patients according to stroke site and compared the incidence of laterality between patient groups.

\section{MATERIALS AND METHODS}

\section{Subjects}

A total of 92 patients (59 men and 33 women) with stroke, who were referred to Department of Rehabilitation Medicine at Asan Medical Center for an evaluation of dysphagia, were enrolled in this study. The patients with stroke had definite brain lesions on computed tomography (CT) or MRI, and data were interpreted by radiology specialists. Patients were divided into two groups: group I patients had supratentorial lesions and group II patients had infratentorial lesions. Patients with a history of dysphagia, dental problems that could affect swallowing function, previous cerebrovascular damage, or pregnancy were excluded. A VFSS was performed on all patients. Patients who could not maintain a sitting posture or could not follow directions and were unable to undergo videofluoroscopy were also excluded.

\section{Methodology}

The oral, pharyngeal, and esophageal stages of swallowing were examined by VFSS using the EasyDiagnost Eleva (Philips, Amsterdam, The Netherlands). Patients were asked to sit in a wheelchair on the right side of the fluoroscope. They were given $5 \mathrm{~mL}$ of thick barium fluid mixture, pureed diet, mechanically altered diet, regular diet, and $5 \mathrm{~mL}$ of thin barium fluid to swallow two times for each diet for the lateral view. Then, laterality was assessed in the anterior-posterior view. Patients were given two spoonfuls of pureed diet and $5 \mathrm{~mL}$ of thin barium fluid to swallow twice. All examinations were recorded as files in the Asan Medical Center database. The study was discontinued if physiatrists involved in the exam decided that the patient was at increased risk of severe aspiration. Fluoroscopic data were analyzed by two physiatrists. Patients were diagnosed with dysphagia if any abnormality in the oral, pharyngeal, or esophageal phases was detected.

The width difference in the barium flow out of the pharyngoesophageal segment was assessed to investigate the presence of laterality $[13,15]$. Laterality was suspected when the flow at the pharyngoesophageal segment was double or more that of the opposite side during two swallows. The difference in flow width in the pharyngoesophageal segment was classified into three categories of right-side dominant in width, left-side dominant in width (LD), and no laterality in width. 


\section{Evaluation}

Patients were evaluated based on the American SpeechLanguage-Hearing Association National Outcome Measurement System (ASHA-NOMS) swallowing scale, which shows dysphagia severity by measuring the diet level with a rating of 1-7 [17] and the clinical dysphagia scale (CDS) which includes items, such as lesion location, tracheostomy, aspiration history, lip sealing, chewing, tongue protrusion, laryngeal elevation, and reflex cough [18]. Oral transit time (OTT), pharyngeal transit time (PTT), premature spillage, residues on the vallecular pouch or pyriform sinus, laryngopharyngeal incoordination, functional dysphagia scale (FDS) $[19,20]$, and penetrationaspiration scale (PAS) [21] were evaluated using VFSS. The OTT was measured from the point when the bolus made the first posterior-ward movement until the head of the bolus reached the back of the tongue. The PTT was measured from the point when the head of the bolus moved beyond the ramus of the mandible until the tail of the bolus passed the cricopharyngeal region $[22,23]$. The OTT and PTT were measured based on solid diet. The FDS was used to assess dysphagia severity and the PAS was used to evaluate the amount of airway invasion. Unlike ASHA-NOMS, higher CDS, FDS, and PAS scores indicate the presence of severe dysphagia.

\section{Statistical analysis}

The Mann-Whitney U-test was used to compare age,
OTT, PTT, PAS, CDS, and FDS between patients who showed laterality and those who did not. The chi-square test was used to compare gender, type, and location of the lesion, presence of motor or facial weakness, presence of laterality, presence of residue in vallecular or pyriform sinuses, presence of oral phase delay, pharyngeal weakness, and presence of aspiration or penetration. The statistical analysis was performed using SPSS ver. 18.0 (SPSS Inc., Chicago, IL, USA). p-values $<0.05$ were considered significant.

\section{RESULTS}

Among the patients with stroke, 72 had supratentorial lesions (group I) and 20 had infratentorial lesions (group II). Only 10 patients (13.9\%) in group I and three (15.0\%) in group II showed laterality. The demographic characteristics are shown in Table 1, and the VFSS results of the groups are shown in Table 2.

No significant differences in age, sex, location of lesion, or type of stroke were found between patients with and without laterality (Table 3 ). Mean patient age was $65.8 \pm 9.9$ years, and $69.2 \%$ (nine patients) were men. Only $15.4 \%$ of patients had hemiplegia, indicating that most patients did not have a severe motor deficit. Furthermore, no differences in the presence of motor or facial weakness were found between the groups.

In addition, lesion location did not differ significantly

Table 1. Demographic characteristics of the study patients

\begin{tabular}{|c|c|c|c|}
\hline & Supratentorial $(n=72)$ & Infratentorial $(\mathbf{n}=\mathbf{2 0})$ & p-value \\
\hline Age (yr) & $66.0 \pm 11.7$ & $70.2 \pm 9.8$ & 0.14 \\
\hline Gender (male) & $46(63.9)$ & $13(65.0)$ & 0.93 \\
\hline Laterality & $10(13.9)$ & $3(15.0)$ & 1.00 \\
\hline Brain lesion side (right) & $37(51.4)$ & $13(65.0)$ & 0.32 \\
\hline Stroke type (infarct) & $57(79.2)$ & $20(100)$ & $0.03^{*}$ \\
\hline Presence of motor weakness & & & 0.16 \\
\hline Right & $33(45.8)$ & $14(70.0)$ & \\
\hline Left & $31(43.1)$ & $5(25.0)$ & \\
\hline Presence of hemiplegia & $19(26.4)$ & $2(10.0)$ & 0.52 \\
\hline Presence of facial weakness & $42(58.4)$ & $8(40.0)$ & 0.31 \\
\hline Right & $22(30.6)$ & $5(25.0)$ & \\
\hline Left & $20(27.8)$ & $3(15.0)$ & \\
\hline
\end{tabular}

Values are presented as mean \pm standard deviation or number (\%).

No significant differences were detected in any parameter between the groups.

${ }^{*} \mathrm{p}<0.05$. 
Table 2. Comparison of swallowing functions according to lesion location

\begin{tabular}{lccc}
\hline & Supratentorial $(\mathbf{n}=\mathbf{7 2})$ & Infratentorial $(\mathbf{n = 2 0})$ & p-value \\
\hline Oral transit time, solid (s) & $0.6 \pm 0.7$ & $0.4 \pm 0.1$ & 0.44 \\
\hline Pharyngeal transit time, solid (s) & $0.4 \pm 0.4$ & $0.4 \pm 0.1$ & 0.68 \\
\hline Vallecular pouch residue & $13(18.1)$ & $4(20.0)$ & 1.00 \\
\hline Pyriform sinus residue & $13(18.1)$ & $2(10.0)$ & 0.51 \\
\hline Clinical dysphagia scale & $32.3 \pm 12.6$ & $29.2 \pm 9.88$ & 0.32 \\
Functional dysphagia scale & $3.4 \pm 6.0$ & $2.8 \pm 5.5$ & 0.69 \\
\hline ASHA NOMS & $5.8 \pm 1.6$ & $5.5 \pm 1.4$ & 0.49 \\
PAS $\geq 6$ & $9(12.5)$ & $2(10.0)$ & 0.82 \\
Presence of solid aspiration & $2(2.8)$ & $0(0.0)$ & 0.69 \\
Presence of solid penetration & $5(6.9)$ & $2(10.0)$ & 0.69 \\
\hline Presence of fluid aspiration & $8(11.1)$ & $2(10.0)$ & 0.67 \\
\hline Presence of fluid penetration & $18(25.0)$ & $2(10.0)$ & 0.67 \\
\hline
\end{tabular}

Values are presented as mean \pm standard deviation or number (\%).

No significant differences were detected in any parameter between the groups.

ASHA NOMS, American Speech-Language-Hearing Association National Outcome Measurement System swallowing scale; PAS, penetration-aspiration scale.

${ }^{*} \mathrm{p}<0.05$.

Table 3. Demographic characteristics of the patients with laterality

\begin{tabular}{|c|c|c|c|}
\hline & Laterality $(n=13)$ & No laterality $(n=79)$ & p-value \\
\hline Age (yr) & $65.8 \pm 9.9$ & $67.1 \pm 11.6$ & 0.71 \\
\hline Gender (male) & $9(69.2)$ & $50(63.3)$ & 0.76 \\
\hline Location of lesion & & & 1.00 \\
\hline Supratentorial lesion & $10(76.9)$ & $62(78.5)$ & \\
\hline Infratentorial lesion & $3(23.1)$ & $17(21.5)$ & \\
\hline Brain lesion side (right) & $9(69.2)$ & $41(51.9)$ & 0.37 \\
\hline Stroke type (infarct) & $11(84.6)$ & $66(83.5)$ & 1.00 \\
\hline Presence of motor weakness & & & 0.56 \\
\hline Right & $5(38.5)$ & $42(53.2)$ & \\
\hline Left & $6(46.2)$ & $30(38.0)$ & \\
\hline Presence of hemiplegia & $2(15.4)$ & $19(23.9)$ & 0.59 \\
\hline Presence of facial weakness & & & 0.14 \\
\hline Right & $2(15.4)$ & $25(31.6)$ & \\
\hline Left & $6(46.2)$ & $17(21.5)$ & \\
\hline
\end{tabular}

Values are presented as mean \pm standard deviation or number (\%).

No significant differences were detected in any parameter between the groups.

${ }^{*} \mathrm{p}<0.05$.

between patients who did and did not have laterality. Nine patients $(69.2 \%)$ with laterality had lesions on the right side, whereas four patients $(30.8 \%)$ had lesions on the left side. Laterality occurred only in the left side of patients, indicating that laterality did not correlate with the side of the lesion.

The VFSS results were compared between patients with and without laterality. Parameters, such as OTT, PTT, presence of residue in vallecular or pyriform sinus, PAS, presence of aspiration or penetration, CDS, and ASHA- 
Table 4. Comparison of swallowing functions according to laterality

\begin{tabular}{lccc}
\hline & Laterality $(\mathbf{n}=\mathbf{1 3})$ & No laterality $(\mathbf{n}=\mathbf{7 9})$ & p-value \\
\hline Oral transit time, solid (s) & $0.4 \pm 0.1$ & $0.6 \pm 0.7$ & 0.45 \\
\hline Pharyngeal transit time, solid (s) & $0.4 \pm 0.1$ & $0.4 \pm 0.1$ & 0.26 \\
\hline Vallecular pouch residue & $3(23.1 \%)$ & $14(17.7 \%)$ & 0.70 \\
\hline Pyriform sinus residue & $4(30.8 \%)$ & $11(13.9 \%)$ & 0.21 \\
\hline Clinical dysphagia scale & $28.1 \pm 9.5$ & $32.2 \pm 12.5$ & 0.23 \\
\hline Functional dysphagia scale & $6.7 \pm 7.2$ & $2.7 \pm 5.51$ & $0.02^{*}$ \\
\hline ASHA NOMS & $5.1 \pm 1.8$ & $5.9 \pm 1.5$ & 0.09 \\
\hline PAS $\geq 6$ & $3(23.1 \%)$ & $8(10.1 \%)$ & 0.09 \\
\hline Presence of solid aspiration & $1(7.7 \%)$ & $1(1.3 \%)$ & 0.16 \\
\hline Presence of solid penetration & $2(15.4 \%)$ & $5(6.3 \%)$ & 0.16 \\
\hline Presence of fluid aspiration & $3(23.1 \%)$ & $7(8.9 \%)$ & 0.31 \\
\hline Presence of fluid penetration & $3(23.1 \%)$ & $22(27.8 \%)$ & 0.31 \\
\hline
\end{tabular}

Values are presented as mean \pm standard deviation or number (\%).

No significant differences were detected in any parameter between the groups.

ASHA NOMS, American Speech-Language-Hearing Association National Outcome Measurement System swallowing scale; PAS, penetration-aspiration scale.

${ }^{*} \mathrm{p}<0.05$.

NOMS are shown in Table 4. No significant differences were observed between the groups in these parameters, except the FDS.

\section{DISCUSSION}

The mechanism of swallowing in patients with stroke and dysphagia is currently a focus of much investigation. Dysphagia frequently occurs after hemispheric stroke. Previous studies involving transcranial magnetic stimulation of pharyngeal muscles showed that hemispheric representation of swallowing is bilateral and asymmetric, which explains the variability of dysphagia occurrence after stroke [24]. In particular, the anterolateral frontal and caudolateral precentral cortex are thought to be related to swallowing control [25]. In another study, dysphagia was associated with stroke severity and with large lesions affecting the frontal and insular cortices [26]. Dysphagia occurs more frequently in hemispheric strokes than in brainstem strokes, the latter accounting for only $6 \%$ of dysphagia cases.

The mechanism underlying the neural control of swallowing has not been fully elucidated. Many studies have been conducted to determine the mechanism of swallowing function and dysphagia. The risk of dysphagia increases with disruption of cortical-cortical and cortical- subcortical white matter connections [5]. Hamdy et al. [27] proposed the existence of functional asymmetry of swallowing between the two hemispheres and suggested that damage to the hemisphere containing the dominant pharyngeal cortical center may lead to oropharyngeal dysphagia. This finding implies that if the dominant center is located in the unaffected hemisphere, dysphagia may not occur. He also suggested that dysphagia is more likely to occur after damage to the right hemisphere. Mosier et al. [28] reported that lesions to the left periventricular white matter are more disruptive to swallowing function than lesions to the right. Most patients with long-term dysphagia have left-hemisphere lesions, which agrees with functional MRI studies, suggesting that the left hemisphere dominates the cortical representation of swallowing. The results of these studies are controversial; thus, we divided our patients into two groups according to the lesion location under the hypothesis that lesion location is related to the occurrence of dysphagia.

Khedr et al. [24] found that esophageal muscle control in patients with hemispheric stroke is similar for both hemispheres. Stimulating the unaffected hemisphere evokes a larger cortical map area in non-dysphagic subjects than in dysphagic subjects and the affected hemisphere produces little response in both groups. This finding suggests that dysphagia and stroke lateralization 
may not be related and that the esophagus may be under bilateral motor control.

Whether dysphagia occurs regardless of the affected hemisphere remains to be elucidated. A previous study showed that patients without dysphagia have extensive bilateral pericentral activation in the early subacute phase after stroke when compared to healthy subjects and that lateralization to the contralesional hemisphere is not significantly pronounced [29]. This finding may explain why no significant difference was seen between the two groups in terms of site of lesion and lateralization in our study.

Pathways underlying swallowing functions can be disrupted but rapid recovery can be achieved by bilateral innervation and alternative innervation routes. Motor representation of the pharynx in the unaffected hemisphere increases in patients who recover from dysphagia, whereas patients who do not recover from dysphagia show no changes in cortical motor representation [30]. This observation suggests that reorganization is necessary for patients with stroke to regain normal swallowing function. Subsequent over-activation of the contralesional hemisphere occurs after acute under-activation. This can be followed by normalization of activation in the chronic stage in patients with stroke who suffer from dysphagia [29]. Recovery of dysphagia occurs rapidly with plastic changes on the healthy side [31]. This may account for why laterality did not frequently occur in patients in our study. The FDS is a quantifiable method to assess dysphagia severity [20], and our FDS results were significantly different between patients with and without laterality (Table 4). This finding suggests that patients with laterality showed more severe dysphagia symptoms than those without laterality, who may have employed compensatory pathways that mitigated the symptoms of dysphagia.

In addition, when lesions are large and invade the primary motor cortex, all swallowing-related cortical areas may be disrupted, resulting in long-term dysphagia [2]. A subgroup analysis of patients with large lesions may show a higher incidence of dysphagia; however, this was not investigated in this study.

Although some studies have shown evidence of laterality, the existence of laterality remains controversial. Swallowing laterality has been studied in healthy subjects, but studies on patients with stroke and dysphagia are rare.
Kim et al. [15] reported laterality in about $40 \%$ of patients with hemiplegic stroke but the direction of laterality did not correlate with the direction of hemiplegia. Swallowing laterality occurred more frequently on the right side, which differs from our findings of laterality on the left side. In this study, many hemiplegic patients did not show swallowing laterality; therefore, it was insufficient to conclude the existence of a relationship between laterality and stroke.

Seta et al. [13] reported that swallowing dominance is more prevalent on the LD in both sexes and that the percentage of $L D$ in men tends to increase with age. These authors reported laterality with a LD pattern in $42 \%$ of subjects, with approximately $70 \%$ of women showing no laterality whereas $70 \%$ of men $>51$ years of age had LD in passage. In agreement with those results, patients in our study showed laterality on the LD. In our study, laterality did not seem to be influenced by the stroke itself and may have existed prior to the onset of stroke. The upper esophagus is located slightly on the left side from the midline due to compression by the trachea and vertebral bodies [32], and this anatomical relationship may have caused laterality in our patients.

There are several limitations to our study. First, the patients with stroke were not grouped according to the severity and extent of lesions. As large lesions involving swallowing-related cortical areas could induce dysphagia symptoms, the extent of the stroke lesions should be considered in the future. Second, we could not identify patients who had lesions on corticobulbar tracts. Patients with corticobulbar lesions may have more severe dysphagic symptoms compared to patients with lesions elsewhere. Third, we could not assess the presence of swallowing laterality before the onset of stroke. Fourth, there was a relatively smaller sample size in the second group when compared with the first group. Post-stroke dysphagic patterns show differences according to location of the brain lesion, whether it was a supratentorial or infratentorial lesion $[6,16]$, unlike our study which showed no differences between supratentorial or infratentorial lesions. This may have been due to the discrepancy in the sample size between the two groups.

Our study investigated the presence of laterality in patients with stroke with the aim of identifying whether factors, such as lesion location or motor weakness, correlate with the occurrence of laterality. Laterality was shown in 
a small number of patients but was not prevalent in the patients enrolled in our study. Our results suggest that lesion location does not impact the incidence of swallowing laterality. Therefore, we cannot elucidate the usefulness of dysphagia compensatory techniques, such as head rotation and head tilt. Further studies with a larger sample size are needed to confirm our results.

In conclusions, our results suggest that laterality was not prevalent among patients with stroke and that lesion side, location of the brain lesion, or presence of motor weakness did not strongly influence swallowing laterality. Although stroke can cause symptoms of dysphagia, it is difficult to conclude that stroke has a crucial impact on swallowing laterality. Further studies with a larger sample size will be needed in the future.

\section{CONFLICT OF INTEREST}

No potential conflict of interest relevant to this article was reported.

\section{REFERENCES}

1. Meng NH, Wang TG, Lien IN. Dysphagia in patients with brainstem stroke: incidence and outcome. Am J Phys Med Rehabil 2000;79:170-5.

2. Falsetti P, Acciai C, Palilla R, Bosi M, Carpinteri F, Zingarelli A, et al. Oropharyngeal dysphagia after stroke: incidence, diagnosis, and clinical predictors in patients admitted to a neurorehabilitation unit. J Stroke Cerebrovasc Dis 2009;18:329-35.

3. Bussell SA, Gonzalez-Fernndez M. Racial disparities in the development of dysphagia after stroke: further evidence from the Medicare database. Arch Phys Med Rehabil 2011;92:737-42.

4. Barer DH. The natural history and functional consequences of dysphagia after hemispheric stroke. J Neurol Neurosurg Psychiatry 1989;52:236-41.

5. Cola MG, Daniels SK, Corey DM, Lemen LC, Romero $\mathrm{M}$, Foundas AL. Relevance of subcortical stroke in dysphagia. Stroke 2010;41:482-6.

6. Park DH, Chun MH, Lee SJ, Song YB. Comparison of swallowing functions between brain tumor and stroke patients. Ann Rehabil Med 2013;37:633-41.

7. Martino R, Foley N, Bhogal S, Diamant N, Speechley M, Teasell R. Dysphagia after stroke: incidence, diagnosis, and pulmonary complications. Stroke 2005;36:2756-63.

8. Humbert IA, Robbins J. Dysphagia in the elderly. Phys Med Rehabil Clin N Am 2008;19:853-66, ix-x.

9. Langdon PC, Lee AH, Binns CW. Dysphagia in acute ischaemic stroke: severity, recovery and relationship to stroke subtype. J Clin Neurosci 2007;14:630-4.

10. Kim SY, Kim TU, Hyun JK, Lee SJ. Differences in videofluoroscopic swallowing study (VFSS) findings according to the vascular territory involved in stroke. Dysphagia 2014;29:444-9.

11. Kim HM, Choi KH, Kim TW. Patients' radiation dose during videofluoroscopic swallowing studies according to underlying characteristics. Dysphagia 2013;28:153-8.

12. Bhattacharyya N, Kotz T, Shapiro J. The effect of bolus consistency on dysphagia in unilateral vocal cord paralysis. Otolaryngol Head Neck Surg 2003;129:632-6.

13. Seta $H$, Hashimoto $K$, Inada $H$, Sugimoto A, Abo M. Laterality of swallowing in healthy subjects by AP projection using videofluoroscopy. Dysphagia 2006;21:191-7.

14. Mosier KM, Liu WC, Maldjian JA, Shah R, Modi B. Lateralization of cortical function in swallowing: a functional MR imaging study. AJNR Am J Neuroradiol 1999;20:1520-6.

15. Kim MS, Lee SJ, Kim TU, Seo DH, Hyun JK, Kim JI. The influence of laterality of pharyngeal bolus passage on dysphagia in hemiplegic stroke patients. Ann Rehabil Med 2012;36:696-701.

16. Lee CK, Kim JA. Pattern of post-stroke swallowing disorder according to the brain lesion. J Korean Acad Rehabil Med 2001;25:193-201.

17. Kim J, Oh BM, Lee GJ, Lee SA, Chun SW, Han TR. Clinical factors associated with severity of post-stroke dysphagia. Brain Neurorehabil 2011;4:116-20.

18. Han TR, Paik NJ, Park JW. The clinical functional scale for dysphagia in stroke patients. Korean J Stroke 2001;3:153-7.

19. Han TR, Paik NJ, Park JW. Quantifying swallowing function after stroke: a functional dysphagia scale based on videofluoroscopic studies. Arch Phys Med Rehabil 2001;82:677-82.

20. Han TR, Paik NJ, Park JW. The functional dysphagia scale using videofluoroscopic swallowing study in stroke patients. J Korean Acad Rehabil Med 
1999;23:1118-26.

21. Rosenbek JC, Robbins JA, Roecker EB, Coyle JL, Wood JL. A penetration-aspiration scale. Dysphagia 1996;11:93-8.

22. Lin CW, Chang YC, Chen WS, Chang K, Chang HY, Wang TG. Prolonged swallowing time in dysphagic Parkinsonism patients with aspiration pneumonia. Arch Phys Med Rehabil 2012;93:2080-4.

23. Martin BJ, Corlew MM, Wood H, Olson D, Golopol LA, Wingo $\mathrm{M}$, et al. The association of swallowing dysfunction and aspiration pneumonia. Dysphagia 1994;9:1-6.

24. Khedr EM, Abo-Elfetoh N, Ahmed MA, Kamel NF, Farook M, El Karn MF. Dysphagia and hemispheric stroke: a transcranial magnetic study. Neurophysiol Clin 2008;38:235-42.

25. Alberts MJ, Horner J, Gray L, Brazer SR. Aspiration after stroke: lesion analysis by brain MRI. Dysphagia 1992;7:170-3.

26. Broadley S, Croser D, Cottrell J, Creevy M, Teo E, Yiu D, et al. Predictors of prolonged dysphagia following acute stroke. J Clin Neurosci 2003;10:300-5.
27. Hamdy S, Aziz Q, Rothwell JC, Crone R, Hughes D, Tallis RC, et al. Explaining oropharyngeal dysphagia after unilateral hemispheric stroke. Lancet 1997;350:68692.

28. Mosier KM, Liu WC, Maldjian JA, Shah R, Modi B. Lateralization of cortical function in swallowing: a functional MR imaging study. AJNR Am J Neuroradiol 1999;20:1520-6.

29. Teismann IK, Suntrup S, Warnecke T, Steinstrater O, Fischer M, Floel A, et al. Cortical swallowing processing in early subacute stroke. BMC Neurol 2011;11:34.

30. Hamdy S, Aziz Q, Rothwell JC, Power M, Singh KD, Nicholson DA, et al. Recovery of swallowing after dysphagic stroke relates to functional reorganization in the intact motor cortex. Gastroenterology 1998;115:1104-12.

31. Hamdy S, Rothwell JC, Aziz Q, Singh KD, Thompson DG. Long-term reorganization of human motor cortex driven by short-term sensory stimulation. Nat Neurosci 1998;1:64-8.

32. Schmalfuss IM, Mancuso AA, Tart RP. Postcricoid region and cervical esophagus: normal appearance at CT and MR imaging. Radiology 2000;214:237-46. 